\title{
Una revisión sistemática: Actitud hacia la investigación en universidades de Latinoamérica
}

\section{A systematic review: Attitude towards research in Latin American universities}

Lina Iris Palacios Serna $\underline{0000-0001-5492-3298}$

Universidad Privada Antenor Orrego, Perú.

1palacioss1@upao.edu.pe
Recibido: 26/05/2021

Aprobado: 16/08/2021

Publicado: 15/09/2021

\section{Resumen}

El objetivo del presente estudio fue señalar la evidencia científica disponible sobre la actitud hacia la investigación, a partir de la revisión sistemática de artículos relevantes existentes en la literatura científica. El análisis sistemático de estos estudios, muestra que el $50 \%$ de la población de estudio presenta una actitud favorable hacia la investigación, el $41.7 \%$ muestra una actitud desfavorable y solo el $8.3 \%$ medianamente favorable.Asimismo, en relaciónalos componentes, se aprecia que el componente cognitivo muestra una categoría positiva con un $35.7 \%$, mientras que el componente afectivo, presenta una categoría positiva con un $21.4 \%$ a comparación de la categoría negativa, que se encuentra en un $14.2 \%$. Mientras que el componente conductual muestra una categoría positiva con un $21.4 \%$ en relación a la categoría negativa que presentó un 7.1\%. Por ello, es relevante cubrir con la necesidad de motivación y uso de estrategias por parte de los docentes responsables de los cursos en investigación para promover la actitud hacia la investigación en estudiantes de pregrado y posgrado.

Palabras clave: actitud; investigación; Latinoamérica; revisión sistemática.

\begin{abstract}
Abstrac
The objective of this research was to indicate the available scientific evidence on the attitude towards research, based on the systematic review of relevant articles in the scientific literature. From a total of 108 initial references detected, 09 researches were selected which fulfilled the defined quality and selection criteria. The systematic analysis of these researches seems to indicate that $50 \%$ of the research population has a favorable attitude towards research, $41.7 \%$ shows an unfavorable attitude and only $8.3 \%$ moderately favorable. Likewise, in the case of the components, the cognitive component shows a positive category with $35.7 \%$, while the affective component has a positive category with $21.4 \%$ compared to the negative category, which is $14.2 \%$. While the behavioral component shows a positive category with $21.4 \%$ in relation to the negative category that presented $7.1 \%$. This refers to the need for motivation and use of strategies by teachers of research courses to promote the attitude towards research in undergraduated and graduated students.
\end{abstract}

Keywords: attitude; research; Latin America; systematic review. 


\section{Introducción}

La investigación es una práctica que está determinada por condiciones que propicien el involucramiento y el deseo por perfeccionar y apoyar el conocimiento científico, explorar los vacíos y contribuir con mejoras significativas frente a los resultados de investigación. De acuerdo a la competencia investigativa, es una exigencia importante dentro del ámbito académico y parte de los requerimientos en todos los niveles formativos, tanto en pregrado, posgrado y posdoctoral. No obstante, en cada nivel académico, la complejidad y exigencia es distinta. Por tanto, en ocasiones, el desarrollo de la investigación se ve limitada por la por falta de conocimiento sobre las exigencias del estricto rigor científico, desconocimiento de la metodología, inadecuado manejo del marco teórico, entre otros.

La variable en estudio parte de un primer aspecto importante, la actitud, la cual, según Morris y Maisto (2005), refieren que es una estructura que se muestra parcialmente estable que contiene creencias, sentimientos y conducta frente a un determinado objeto. Por lo que las creenciaspensamientos permitirán el conocimiento y el grado de exploración de un objeto, mientras que los sentimientos permitirán mostrar la sensaciones positivas o negativas $\mathrm{y}$, por tanto, la conducta orientará la acción a tomar frente al objeto. A la vez según Allport (1935), la actitud viene a ser un estado mental y neurológico que se organiza a partir de la práctica, ejerciendo gran influencia de manera activa sobre el comportamiento del ser humano para poder responder a su contexto. Asimismo, Motta (2009) refiere que la actitud es la forma positiva o negativa de responder frente a un objeto, hecho, etc., el cual se determina por las creencias, sentimientos y conductas interactúan de manera interdependiente en este proceso.

Es así que, para Valverde (2005), la actitud investigadora es aquella posición personal que permite actuar y/o participar en la investigación de acuerdo a las capacidades, experticia, características, habilidades como investigador, que llevan a desarrollar la postura investigadora frente a sus conocimientos previos, dominios, especialidad etc.

Sin embargo, tomando en cuenta lo explicado por Rojas y Méndez (2013), destacan que los estudiantes, por naturaleza, presentan la inquietud y capacidad exploratoria frente a su realidad, la que debe estar orientada a la motivación por asumir la participación dedicada, interactiva y participativa dentro de los procesos investigativos durante su formación en pregrado. En este sentido, Rojas et al. (2012) destacan que la actitud hacia la investigación, desde la pedagogía, es la predisposición positiva del estudiante universitario que facilita el aprendizaje adecuado de los procesos de investigación. Por ello, es importante que el desarrollo de las capacidades y la predisposición para la investigación sean aspectos importantes a estimular desde los primeros ciclos de formación académica, a fin de crear mejores formas de aprendizaje frente al desarrollo y producción científica.

Sobre lo expuesto, la actitud para la investigación vendría a ser la predisposición del sujeto frente a la búsqueda y trasmisión de conocimiento, lo que parte de factores predisponentes personales y aspectos académicos. Los factores predisponentes personales hacen referencia a la capacidad cognitiva, afectiva y conductual; de ahí la concepción de los diversos autores por mencionar que es una predisposición o estado mental para actuar.

No obstante, tener actitud frente a la investigación, hace referencia a la complementación de las condiciones internas del sujeto con las condiciones de soporte externo, lo cual resulta en el deseo por indagar, conocer y ampliar el conocimiento; pero no solo por normatividad, sino por soporte gerencial de las estructuras de las instituciones educativas superiores en brindar espacios y apoyo económico para efectivizar una investigación. En cuanto a la normatividad peruana, la Ley Universitaria $\mathrm{N}^{\circ} 30220$ (2014), señala que la investigación "constituye una función esencial y obligatoria de la universidad, y esta debe responder a través de la producción de conocimientos y el desarrollo de tecnologías que respondan a las necesidades de la sociedad" (p. 7). Al respecto, Cervantes et al. (2019), hace mención que la Ley n. ${ }^{\circ} 30220$ resalta con gran interés la investigación, sin embargo, no toma en cuenta las condiciones actuales de las universidades en relación a la implementación y desarrollo de la investigación, tecnología e innovación.

Asimismo, Cabrera et al. (2011) plantean que, para mejorar la calidad investigativa, se deben realizar profundos cambios desde el contenido 
curricular y la inclusión de una secuencia lógica en las asignaturas, lo cual apoye el interés por la investigación para que los estudiantes vayan obteniendo las competencias necesarias que le permitan finalmente realizar investigaciones de calidad. Ante ello, Guzmán (2018) recomienda que es importante que "... se induzca a los estudiantes en las diversas asignaturas a realizar trabajos de investigación con diferentes grados de complejidad dependiendo del nivel de los estudiantes" (p. 78).

Por otro lado, Aldana y Joya (2011) refieren que el contexto resulta importante para lograr la actitud consciente frente a la construcción del conocimiento y la forma de investigar, requiriéndose de una estructuración de los planes de estudio y que los docentes realicen un acompañamiento al estudiante durante el proceso investigativo.

De igual modo, Ruiz y Torres (2005) señalan que existen otros factores que también van a influir directamente en el proceso deenseñanza aprendizaje en investigación, como son: el paradigma científico imperante, la cultura organizacional investigativa, el clima organizacional y las estrategias de enseñanza. No obstante, Giraldo (2010) señala que coexisten, además, ciertos obstáculos para la formación de una actitud investigativa en la universidad, como: (1) la didáctica en metodología de la investigación, (2) falta de políticas claras sobre investigación, (3) falta de infraestructura que genere espacios para la realización de la investigación (4) escaso financiamiento.

Cabe mencionar que la U.S. News and World Report (2012), citado por De La Cruz y Rodríguez (2019), explica que la investigación siempre ha sido una actitud constante en la vida del hombre, por lo que fundamenta los nuevos conocimientos sobre la realidad en la cual viven; frente a ello se beneficia la población y el avance de la sociedad. El progreso a través de la investigación, es una práctica importante la cual se denomina "economía basada en el conocimiento", la cual es muy bien practicada por Estados Unidos (EE. UU), país que llega a invertir más del $2.5 \%$ de su producto interno bruto (PBI) solo en actividades científicas, promoviendo así el desarrollo tecnológico y competitividad. Otro ejemplo es el país de Europa, Finlandia y Suecia, los que disponen de un 3.5\% del PBI en investigación y Perú solo el $0.15 \%$. Frente a la pobre inversión en investigación en el Perú, Almeida (2019) refiere que no se invierte lo suficiente en investigación y desarrollo en comparación con otros países de Latinoamérica, pues solo se gasta el $0,08 \%$ del PBI, mientras que en Colombia $(0,25 \%)$, en Chile $(0,38 \%)$ y Brasil $(1,24 \%)$.

Consecuentemente, es posible que la falta de inversión en investigación, limite la iniciativa y la continuidad por investigar, pues no se aprecia el adecuado soporte económico para viabilizar proyectos e investigaciones relacionadas con el desarrollo e innovación en la sociedad. Ante esto, si se comprendiera que el desarrollo económico de una comunidad se basa en la investigación, quizás las perspectivas de inversión fueran diferentes.

Por otro lado, De La Cruz y Rodríguez (2019) señalan que las personas dedicadas a la investigación en el Perú, en relación a otros países, es menor, representando el $0.01 \%$ del total de la población, teniendo como visión la necesidad de promover actividades que permitan desarrollar la investigación en las universidades, más aún en las privadas donde el número de investigadores es menor.

Asimismo, De La Cruz y Rodríguez (2019) destacan que la investigación debe ser una filosofía institucional, la cual esté presente en la formación académica inicial, donde se implemente en las asignaturas la importancia de la investigación para la formación temprana de talentos que tengan el interés y motivación por investigar y valorar la producción de conocimiento que contribuya al avance científico. Al respecto cabe tomar en consideración lo mencionado por Muñoz (2011), quien explica que "se exige que los egresados presenten un trabajo de titulación con ciertas características y rigor científico, pero no se les prepara de manera suficiente para elaborarlo" ( $p$. 39). En este sentido, no basta contemplar en el plan de estudios las asignaturas de tesis al final de los últimos ciclos, sino se debe emplear estrategias en los estudiantes para que puedan realizar un trabajo de denotada calidad científica.

Por lo explicado, es importante que las universidades privadas del Perú y de Latinoamérica puedan promover investigaciones de alto nivel, que les permita apreciar en la investigación una oportunidad para posicionarlas dentro del campo del desarrollo e innovación, en donde los conocimientos producidos en ellas, sean insumos para investigaciones futuras en otros países. 
Por otro lado, en la misma realidad, la producción científica es precaria, tal es así que en el Informe de SJR Scimago Journal \& Country Rank (2018) se mostró que la producción científica en el ámbito de la educación, en el año 2017 América Latina, presentó 3130, cifra que es considerada baja en relación a la producción mundial que muestra un $5,4 \%$, en comparación de Perú, en donde la producción de 59 artículos en educación representó el $0,10 \%$ de la producción mundial.

Sobre lo expuesto, existe una concatenación de hechos necesarios para promover la cultura investigativa, desde la promoción de la actitud por investigar, hasta la motivación por investigar y el espíritu para contribuir ante la necesidad del desarrollo e innovación de la sociedad. Por ello, la investigación por ser potencial en los jóvenes, debería promoverse desde los primeros ciclos de formación académica, para generar actitudes de identificación e involucramiento en la investigación. De ahí el requerimiento de docentes idóneos para este proceso formativo inicial en investigación en los jóvenes, quienes deben cumplir con los estándares de calidad exigidos en la investigación, desarrollo e innovación. A la vez los estudiantes de posgrado deben impulsar ideas claras que fomenten contribuciones y mejoras, ofreciendo soluciones ante problemáticas en particular y no elaborar investigaciones solo por cumplimiento con las exigencias formativas. Los jóvenes inmersos en el estudio, es una población que puede traer beneficios importantes para la 198 investigación, quienes pueden adquirir la experticia poder discriminar, valorar, inferir sobre el contexto en el que se encuentran inmersos. Lo importante es crear el interés por avanzar en los conocimientos, produciendo y comunicando los resultados a la comunidad. La didáctica en investigación plantea la necesidad de motivar actitudes investigativas tanto en los jóvenes de pregrado, como los que llevan estudios avanzados.

Respecto a las actitudes hacia la investigación, Veliz (2019) en su investigación realizada con 142 estudiantes de posgrado de dos universidades de Perú, encontró que la actitud hacia la investigación predomina el nivel Regular $(1=61,6 \%$ y 2 $=57,14 \%$ ). Así mismo en los componentes cognoscitivo, afectivo y conductual los estudiantes de posgrado de universidades en estudio mostraron mayor tendencia en el nivel regular.
Asimismo, Meza (2019) realizó una investigación con 193 estudiantes de los últimos ciclos, encontrando que el $54,8 \%$ de los estudiantes de posgrado evidencian una categoría regular con $45,2 \%$ en la actitud positiva hacia la investigación. Así mismo en los componentes afectivo y conductual muestran categoría regular, mientras que el componente cognitivo presento una categoría positiva.

Al respecto, Cervantes et al. (2019), a modo reflexivo, mencionan que resulta de vital importancia la revisión y evaluación de contenidos relacionados con las asignaturas que desarrollan investigación, además de revisar la idoneidad de los docentes a cargo, pues señalan que, en estudios realizados sobre actitud hacia la investigación en estudiantes de pregrado, de presenta baja tendencia por percibirla como tediosa y mostrando rechazo e indiferencia.

Ante lo expuesto, la presente revisión centra su objetivo en señalar la evidencia científica disponible sobre la actitud hacia la investigación, a partir de la revisión sistemática de artículos relevantes existentes en la literatura científica.

\section{Metodología}

El diseño empleado en el presente artículo es revisión sistemática, en donde se logró analizar y sistematizar la información como principal evidencia de los resultados presentados en cada uno de los artículos en torno a la temática de actitud hacia la investigación.

\section{Proceso de selección:}

Los artículos fueron seleccionados de las bases de datos Scielo, Redalyc, Dialnet, Semanticscholar, utilizando como palabras clave: actitud investigativa, actitud hacia la investigación, actitud en investigación, todas ellas en idioma español.

En el presente estudio se tuvieron en cuenta los siguientes criterios de inclusión: artículos publicados en bases Scielo, Redalyc, Dialnet, Semanticscholar, entre los años 2015-2020. Estudiados en población de pregrado y posgrado de universidades de Latinoamérica, en donde la temática principal fue la actitud hacia la investigación. Los estudios incluidos además pasaron por los criterios de calidad de DixonWoods. 




Figura 1. Algoritmo de selección de estudios según criterios de selección.

\section{Codificación de los estudios}

Se tomó en cuenta las siguientes categorías, para la elaboración de la base de datos: (1) autor/es, año de publicación, país. (2) Población de estudio: maestría y posgrado. (3) Diseño. (4) Muestra. (5) Instrumento y (6) principales resultados.

Para el procesamiento de la información, se empleó análisis de contenido tomando en consideración el análisis simultaneo de todos los datos, logrando profundización que permita enriquecer la interpretación (Hernández et al., 2014).

\section{Resultados y discusión:}

De acuerdo a las revisiones de los 09 artículos incluidos realizada se tienen los siguientes resultados:

Tabla 1. Artículos Incluidos Según las Consideraciones de Codificación.

\begin{tabular}{|c|c|c|c|c|c|}
\hline Autor(es)/año/país & $\begin{array}{l}\text { Nombre } \\
\text { Artículo }\end{array}$ & Diseño & Muestra & Instrumento & Resultados \\
\hline $\begin{array}{l}\text { Olivera, E. (2020) } \\
\text { País: Perú }\end{array}$ & $\begin{array}{l}\text { Actitudes hacia } \\
\text { la investigación } \\
\text { de bachilleres en } \\
\text { administración y } \\
\text { psicología de una } \\
\text { universidad } \\
\text { peruana }\end{array}$ & $\begin{array}{l}\text { Cuantitativo - } \\
\text { descriptivo }\end{array}$ & $\begin{array}{l}152 \text { estudiantes } \\
\text { de pregrado ( } 74 \\
\text { Administración } \\
\text { /78 Psicología) } \\
\text { Muestreo } \\
\text { probabilístico }\end{array}$ & $\begin{array}{l}\text { Escala de actitudes } \\
\text { hacia la } \\
\text { investigación } \\
\text { científica de } \\
\text { Portocarrero \& De } \\
\text { la Cruz (2006) } \\
\text { Confiabilidad } \\
\text { general de } 0,70 .\end{array}$ & $\begin{array}{l}\text { Estudiantes de } \\
\text { administración: Actitud } \\
\text { desfavorable } 67 \% \text { actitud } \\
\text { favorable } 33 \% \text {. Estudiantes } \\
\text { de psicología: Actitud } \\
\text { favorable } 51 \% \text { Actitud } \\
\text { desfavorable } 49 \%\end{array}$ \\
\hline
\end{tabular}




\begin{tabular}{|c|c|c|c|c|c|}
\hline $\begin{array}{l}\text { Chara y Olortegui } \\
\text { (2018) } \\
\text { País: Perú }\end{array}$ & $\begin{array}{l}\text { Factores } \\
\text { asociados a la } \\
\text { actitud hacia la } \\
\text { investigación en } \\
\text { estudiantes } \\
\text { universitarios de } \\
\text { enfermería }\end{array}$ & $\begin{array}{l}\text { Descriptivo } \\
\text { correlacional } \\
\text { de corte } \\
\text { transversal }\end{array}$ & $\begin{array}{l}100 \text { estudiantes } \\
\text { de pregrado. } \\
\text { Censo }\end{array}$ & $\begin{array}{l}\text { Escala de Actitudes } \\
\text { hacia la } \\
\text { Investigación } \\
\text { "EACIN" }\end{array}$ & $\begin{array}{l}\text { Actitud hacia la } \\
\text { investigación } \\
\text { Medianamente favorable } \\
(58 \%)\end{array}$ \\
\hline $\begin{array}{l}\text { Rodríguez (2017) } \\
\text { País: Venezuela. }\end{array}$ & $\begin{array}{l}\text { Actitudes hacia } \\
\text { la investigación } \\
\text { en los } \\
\text { participantes de } \\
\text { postgrado de la } \\
\text { universidad } \\
\text { nacional abierta }\end{array}$ & $\begin{array}{l}\text { Transeccional, } \\
\text { comparativo }\end{array}$ & $\begin{array}{l}155 \text { estudiantes } \\
\text { posgrado ( } 75 \\
\text { Maestría en } \\
\text { Administración } \\
\text { educativa/ } 80 \\
\text { Administración } \\
\text { de Negocios) } \\
\text { No especifica }\end{array}$ & $\begin{array}{l}\text { Escala de actitudes } \\
\text { hacia la } \\
\text { investigación. } \\
\text { Componente } \\
\text { cognitivo: índice } \\
\text { de confiabilidad } \\
0,74 \text {. } \\
\text { Componente } \\
\text { afectivo: índice de } \\
\text { confiabilidad } 0,85 \text {. } \\
\text { Componente } \\
\text { conductual: índice } \\
\text { de confiabilidad de } \\
0,80 \text {. }\end{array}$ & $\begin{array}{l}\text { Maestríaen administración } \\
\text { educativa: } \\
\text { Actitud favorable } 8.7 \% \\
\text { Componente cognitivo: } \\
\text { Positivo componente } \\
\text { afectivo: positivo } \\
\text { Componente conductual: } \\
\text { muy positivo. } \\
\text { Maestría de Negocios: } \\
\text { Actitud favorable: } 92.5 \% \\
\text { Componente cognitivo: } \\
\text { Positivo Componente } \\
\text { afectivo: positivo } \\
\text { Componente conductual: } \\
\text { muy positivo. }\end{array}$ \\
\hline $\begin{array}{l}\text { Gálvez et al (2019) } \\
\text { País: Perú }\end{array}$ & $\begin{array}{l}\text { Actitud hacia la } \\
\text { investigación } \\
\text { científica al final } \\
\text { de la carrera de } \\
\text { Enfermería en } \\
\text { Perú }\end{array}$ & $\begin{array}{l}\text { Cuantitativa, } \\
\text { descriptiva, } \\
\text { transversal }\end{array}$ & $\begin{array}{l}80 \text { estudiantes de } \\
\text { pregrado. } \\
\text { Probabilístico }\end{array}$ & $\begin{array}{l}\text { Escala de actitudes } \\
\text { hacia la } \\
\text { Investigación } \\
\text { (EACIN): } \\
\text { Confiabilidad de } \\
0,854 \\
\text { El cuestionario } \\
\text { estructurado: } \\
\text { confiabilidad de } \\
0,752 \text {. }\end{array}$ & $\begin{array}{l}\text { Actitud negativa frente a la } \\
\text { investigación: } 8,8 \% \\
\text { Actitud positiva frente a la } \\
\text { investigación: } 41.3 \% \\
\text { Dimensión Cognitiva: } \\
62,5 \% \text { reconocen que la } \\
\text { investigación científica } \\
\text { permite la construcción del } \\
\text { conocimiento y la } \\
\text { producción del nuevo } \\
\text { saber. } \\
\text { Dimensión Afectiva: } \\
81.2 \% \text { mostraron antipatía } \\
\text { ante la investigación. } \\
\text { Dimensión conductual: } \\
66,3 \% \text { no presentan } \\
\text { disposición para realizar } \\
\text { Investigación científica. }\end{array}$ \\
\hline $\begin{array}{l}\text { Ortega et al. } \\
(2018) \\
\text { País: } \\
\text { Paraguay }\end{array}$ & $\begin{array}{l}\text { Percepción y } \\
\text { actitudes hacia la } \\
\text { investigación } \\
\text { científica. }\end{array}$ & $\begin{array}{l}\text { Observacional, } \\
\text { transversal, } \\
\text { prospectivo y } \\
\text { descriptivo. }\end{array}$ & $\begin{array}{l}358 \text { estudiantes } \\
\text { de pregrado } \\
\text { Probabilístico }\end{array}$ & $\begin{array}{l}\text { El instrumento } \\
\text { utilizado es una } \\
\text { adaptación del IAI } \\
\text { (Incide de Actitud } \\
\text { hacia la } \\
\text { Investigación) } \\
\text { desarrollada por } \\
\text { Rojas, M. y } \\
\text { Méndez, R. (2013) } \\
\text { Índice de } \\
\text { fiabilidad: } 0,871 \text {. }\end{array}$ & $\begin{array}{l}\text { Satisfechos con el nivel } \\
\text { académico y científico de } \\
\text { su carrera: } 60 \% \text { de } 100 \% \\
\text { No se sienten con la } \\
\text { preparación necesaria para } \\
\text { postular proyectos de } \\
\text { investigación: } 40 \% \\
\text { Más del } 90 \% \text { consideran } \\
\text { que la investigación } \\
\text { ayudara a su vida } \\
\text { profesional. } \\
\text { Menos del } 80 \% \text { les gustaría } \\
\text { participar en semilleros de } \\
\text { investigación. }\end{array}$ \\
\hline
\end{tabular}




\begin{tabular}{|c|c|c|c|c|c|}
\hline $\begin{array}{l}\text { Acón et al. } \\
\text { (2015) } \\
\text { País: Costa } \\
\text { Rica }\end{array}$ & $\begin{array}{l}\text { Conocimientos y } \\
\text { actitudes hacia la } \\
\text { investigación } \\
\text { científica en } \\
\text { estudiantes de } \\
\text { medicina de una } \\
\text { Universidad } \\
\text { Privada de Costa } \\
\text { Rica, } 2015\end{array}$ & $\begin{array}{l}\text { Observacional } \\
\text { Analítico } \\
\text { trasversal. }\end{array}$ & $\begin{array}{l}233 \text { estudiantes } \\
\text { de pregrado. } \\
\text { Probabilístico }\end{array}$ & $\begin{array}{l}\text { Cuestionario sobre } \\
\text { conocimientos, } \\
\text { Actitudes y } \\
\text { prácticas creado } \\
\text { Por Díaz-Vélez, C } \\
\text { (7) et al y } \\
\text { Modificado por } \\
\text { Cabrera et al. } \\
\text { No precisa } \\
\text { confiabilidad del } \\
\text { instrumento. }\end{array}$ & $\begin{array}{l}\text { Actitudes en investigación } \\
\text { Inadecuado: } 78.5 \% \\
\text { Adecuado: } 21.5 \%\end{array}$ \\
\hline $\begin{array}{l}\text { Paredes y } \\
\text { Moreta } \\
(2020) \\
\text { País. } \\
\text { Ecuador }\end{array}$ & $\begin{array}{l}\text { Actitudes hacia } \\
\text { la investigación y } \\
\text { autorregulación } \\
\text { del aprendizaje } \\
\text { en los estudiantes } \\
\text { universitario }\end{array}$ & $\begin{array}{l}\text { Comparativo } \\
\text { correlacional }\end{array}$ & $\begin{array}{l}222 \text { estudiantes } \\
\text { de pregrado } \\
\text { No probabilístico }\end{array}$ & $\begin{array}{l}\text { Inventario de } \\
\text { Procesos de } \\
\text { autorregulación del } \\
\text { aprendizaje } \\
\text { (IPAA). La } \\
\text { consistencia } \\
\text { interna: } \mathrm{a}=.854\end{array}$ & $\begin{array}{l}\text { Factor Cognoscitivo: } \\
\text { mayor relevancia } \\
\text { Factor Afecto: Relevancia } \\
\text { estadística significativa }\end{array}$ \\
\hline $\begin{array}{l}\text { Arellano, F., } \\
\text { Hermoza, R., } \\
\text { Elías, M y } \\
\text { Ramírez, M. } \\
\text { (2017) } \\
\text { País: Perú }\end{array}$ & $\begin{array}{l}\text { Actitud hacia la } \\
\text { investigación de } \\
\text { estudiantes } \\
\text { universitarios en } \\
\text { Lima, Perú }\end{array}$ & $\begin{array}{l}\text { Cuantitativo, } \\
\text { observacional, } \\
\text { transversal }\end{array}$ & $\begin{array}{l}194 \text { estudiantes } \\
\text { de pregrado } \\
\text { Muestreo: no } \\
\text { especifica }\end{array}$ & $\begin{array}{l}\text { Instrumento creado } \\
\text { por los autores (no } \\
\text { especifica el } \\
\text { nombre) } \\
\text { Fiabilidad: } 0,65\end{array}$ & $\begin{array}{l}\text { Actitud hacia la } \\
\text { investigación: } \\
\text { Buena: } 18,6 \% \\
\text { Regular:58,2\% } \\
\text { Mala: } 23.2 \%\end{array}$ \\
\hline $\begin{array}{l}\text { Mercado, M } \\
\text { (2017) } \\
\text { País: Perú }\end{array}$ & $\begin{array}{l}\text { Actitudes hacia } \\
\text { la investigación } \\
\text { en los estudiantes } \\
\text { de la carrera de } \\
\text { Medicina } \\
\text { Humana de la } \\
\text { Universidad } \\
\text { Peruana Los } \\
\text { Andes }\end{array}$ & $\begin{array}{l}\text { Observación, } \\
\text { prospectivo, } \\
\text { transversal }\end{array}$ & $\begin{array}{l}94 \text { de pregrado } \\
\text { Muestreo no } \\
\text { probabilístico, } \\
\text { intencional. }\end{array}$ & $\begin{array}{l}\text { Escala para medir } \\
\text { actitudes hacia la } \\
\text { Investigación No } \\
\text { especifica }\end{array}$ & $\begin{array}{l}\text { Actitudes hacia la } \\
\text { investigación: } \\
\text { Cognitiva: adecuada } \\
\text { Afectiva: Adecuada } \\
\text { Conductual: Adecuada }\end{array}$ \\
\hline
\end{tabular}

En la tabla 1, se aprecia que en las revisiones realizadas entre los años 2015-2020, el 55.6\% de los artículos, pertenecen a investigaciones realizadas en el Perú y el $44.4 \%$ en otros países de Latinoamérica.

En cuanto a la actitud hacia a la investigación, según los artículos consultados se presenta similitud en la cantidad de muestra empleada y la percepción que presentan, pues el $50 \%$ presenta actitud favorable ante la investigación, el $41.7 \%$ muestra una actitud desfavorable y solo el $8.3 \%$ medianamente favorable. Asimismo, de acuerdo a los componentes, el componente cognitivo muestra una categoría positiva con un $35.7 \%$, mientras que el componente afectivo, presenta una categoría positiva con un $21.4 \%$. En comparación de la categoría negativa, que se encuentra en un $14.2 \%$. Mientras que el componente conductual muestra una categoría positiva con un $21.4 \%$ en relación a la categoría negativa que presentó un $7.1 \%$.

\section{Discusión}

De acuerdo a los resultados encontrados en la revisión de los artículos, se infiere que el tema de actitud hacia la investigación se está desarrollando progresivamente más en Perú que en otros países (según los países citados); sin embargo, los datos resultan relevantes para poder realizar el análisis respectivo.

En los artículos revisados, se aprecia que la actitud para investigar viene a ser la predisposición por investigar, haciendo uso del juicio crítico, perspectiva, creatividad, innovación etc. Situación que se ve beneficiada gracias a la competencia docente en la formación de los estudiantes en investigación, que implique la enseñanza de capacidades de investigación y llevando a los estudiantes a ser más innovadores en la propuesta y comunicación de sus ideas lo que permita avanzar en el conocimiento científico. Por ello, Olivera (2020) hace referencia que en educación superior 
resulta ser importante potenciar en los estudiantes la actitud hacia la investigación, mediante la preparación de las capacidades para el desarrollo y la producción científica, como eje transversal en el periodo formativo.

Asimismo, los estudios reflejan que los estudiantes muestran una actitud general positiva hacia la investigación, debido a que existen condiciones que permiten el desarrollo y la tecnología, relacionándolas con la participación docente $\mathrm{y}$ el soporte institucional en investigación. De acuerdo a los componentes de la actitud hacia la investigación según los instrumentos de empleados, el componente cognitivo es positivo, mientras que los componentes afectivo y conductual se presentan posiciones divididas entre lo positivo y negativo.

Por otro lado, también se destaca que la existencia del rechazo, indiferencia o aceptación hacia la realización de la investigación, está estrechamente ligada con el factor motivacional mediante el cual se permite indagar de manera proactiva, generando en el componente conductual la búsqueda de soluciones, toma de decisiones, que permitan fortalecer las competencias en investigación. De esta manera, será posible la producción del conocimiento, de examinar críticamente los conocimientos existentes para proponer nuevos conceptos, teorías, etc. fortaleciendo la cultura investigativa.

Ante lo mencionado, cabe destacar lo señalado por Gálvez et al. (2019) quienes hacen referencia que la actitud o predisposición hacia la investigación viene a ser el producto de proceso de formación del estudiante, por lo que la actitud negativa (componente conductual) se relaciona con el escaso manejo de los programas estadísticos para el tratamiento de los datos. Así como también, lo que señalan Paredes y Moreta (2020), quienes explican que la actitud hacia la investigación negativa hará que el estudiante procrastine (componente conductual), evitando iniciar, desarrollar y culminar una investigación. De lo expuesto la actitud investigativa se ve influida por varios factores, lo cual se apoya por lo expuesto por Chara y Olortegui (2018) quienes destacan que las actitudes desfavorables o negativas hacia la investigación se ven influidas por el poco tiempo que se tiene para la lectura e inasistencia a los cursos de investigación, que viene a ser los componentes actitudinales de predisposición hacia la formación en investigación.
Por otro lado, en la literatura revisada se destaca que el hecho de experimentar sensaciones de desagrado frente a la investigación puede bloquear la actitud cognoscitiva, disminuyendo su capacidad crítica, el juicio de valor e inclusive el fortalecimiento del pensamiento crítico. Mientras que las sensaciones de agrado favorecerán la actitud hacia la investigación, pues las condiciones afectivas resultan ser motivadores que impulsan la conducta en la medida que de las características psicológicas se desprende la motivación hacia la consecución de un determinado propósito. Teóricamente, lo mencionado, se apoya por lo señalado por Quispe (2014) quien explica que la actitud hacia la investigación viene a ser la predisposición por investigar-indagar y que proviene de los estados psicológicos internos con la presencia del recurso afectivo, que permitirá la aceptación o rechazo de la investigación.

Asimismo, los autores de los artículos revisados refieren la actitud hacia la investigación debe ser trabajada desde los primeros años de formación académica, pues las actitudes positivas o negativas hacia la investigación dependerán de ciertos factores personales (emociones) y factores externos, como las condiciones de enseñanza aprendizaje para la investigación. Como menciona Acón et al. (2015), es relevante que se fomenten la investigación desde los primeros años de formación de la profesión.

De igual modo, es importante destacar que los docentes requieren contar con las competencias necesarias para ser claros mentores de la formación de estudiantes, mostrando una didáctica flexible y dinámica en el proceso de enseñanza de la investigación, que hagan uso de las estrategias necesarias para la motivación por indagar, innovar y desarrollar nuevas propuestas y que fortalezca el equilibrio de los tres componentes actitudinales. En este sentido, además, se debe emplear la retroalimentación dinámica entre la triada estudiante-docente-universidad para efectivizar la investigación, y el estudiante pueda desarrollar una actitud hacia la investigación positiva, que le permite el desarrollo, innovación, creación de conocimientos y promoción de avances tecnológicos con la calidad que se requiere.

Al respecto, cabe mencionar que Munive (2018) encuentra importante el desarrollo de la investigación como un pilar para el desarrollo y crecimiento de manera sostenible de un país. Por 
ello, es importante la formación de profesionales idóneos que fomenten la actitud positiva hacia la investigación. Sin embargo, lo que observa según su estudio es que puede existir una adecuada actitud hacia la investigación, pero no se involucran en proyectos de investigación por falta de tiempo, escaso estímulo hacia la investigación o escasos profesionales para motivar la publicación en revistas indexadas. Y desde esta perspectiva existe un gran reto para los docentes dedicados a la didáctica en investigación.

Al respecto Barja et al. (2019) menciona que las universidades deben fomentar en docentes y estudiantes la capacitación en el uso de herramientas de soporte como los gestores bibliográficos, cómo acceder, cómo realizar la búsqueda de las fuentes bibliográficas, además del uso del juicio crítico a fin de que promover una adecuada producción de conocimiento sobre una buena base teórica, metodológica y estadística. En definitiva, se debe capacitar tanto en la búsqueda de información especializada como en el análisis crítico e interpretación de dicha información.

Finalmente, Palomino (2017) destacó la importancia actual del desarrollo de la investigación científica, pues permite impulsar y contribuir eficientemente con la ciencia, la tecnología, e innovación para favorecer el progreso urgente en el Perú. Por tal razón, en la actualidad, la actitud hacia la investigación en los estudiantes de pre y posgrado es de vital interés, puesto que, gracias a la investigación consciente, se podrán solucionar problemas y llenar vacíos en investigación desde una didáctica reflexiva y motivadora.

\section{Conclusiones}

Según los artículos revisados, se presentan las siguientes conclusiones:

La actitud hacia la investigación se ve beneficiada gracias a la competencia docente en la formación de los estudiantes, tomando el rol de mentor sobre la base de una retroalimentación constante, impulsando la potencialidad y curiosidad por la investigación.

Se debe potenciar en los estudiantes la innovación y creatividad, dentro de los procesos investigativos siendo capaces de romper los típicos paradigmas y no ser solo repositorios de conocimiento.
El rechazo, la indiferencia o la aceptación hacia la realización de la investigación está estrechamente ligada con el factor motivacional, el cual debe ser trabajado por el docente a fin de promover una actitud positiva hacia la investigación.

La actitud hacia la investigación debe ser trabajada desde los primeros años de formación académica, pues las actitudes positivas o negativas hacia la investigación dependerán de ciertos factores personales (emociones) y factores externos como las condiciones de enseñanza aprendizaje para la investigación.

Si las universidades cuentan con el adecuado soporte económico, se debe implementar la unión con aliados estratégicos que permitan crear redes de apoyo colaborativas en investigación, favoreciendo no solo el trabajo multidisciplinario, sino interinstitucional.

\section{Referencias bibliográficas}

Acón, E., Fonseca, K, Artavia, L. y Galán, E. (2015). Conocimientos y actitudes hacia la investigación científica en estudiantes de medicina de una Universidad Privada de Costa Rica. Revista Cuerpo Médico, 8(4), 217-221. 10.35434/rcmhnaaa.2015.84.161

Aldana, G. y Joya N. (2011). Actitudes hacia la investigación científica en docentes de metodología de la investigación. Tabula Rasa, 14, 296-309. http://www.scielo.org.co/ pdf/tara/n14/n14a12.pdf

Aldana, G., Caraballo, G. y Babativa, D. (2016). Escala para medir actitudes hacia la investigación (EACIN): Validación de contenido y confiabilidad. Revista Aletheia, 8(2), 104-121. https://aletheia.cinde.org.co/ index.php/ALETHEIA/article/view/325

Allport, G. (1935). Attitudes. Handbook of Social Psychology.

Almeida, A. (2019). Investigación y desarrollo $(I+D)$ en el Perú: ¿invertimos lo suficiente? [RPP Noticias]. https://rpp.pe/columnistas/ alexandrealmeida/investigacion-y-desarrolloid-en-el-peru-invertimos-lo-suficientenoticia-1204891 ResearchGate.

Arellano-Sacramento, C., Hermoza-Moquillaza, V., Elías-Podestá, M, y Ramírez-Julca, M. (2017). Actitud hacia la investigación de estudiantes universitarios en Lima, Perú. FEM: Revista de la Fundación Educación Médica, 20(4), 191-197. 10.33588/fem.204.901. 
Barja, J., Otoya, O., Vega, E., Moreno, N. y Loli Ponce, R. (2019). Actitudes hacia la investigación de internos de obstetricia rotantes en un hospital de Lima-Perú. Revista de la Facultad de Medicina Humana, 19(4), 53-59. 10.25176/RFMH.v19i4.2341.

Bernal, N. (2019). Estrategia didáctica para el desarrollo de la actitud investigativa de los estudiantes de ingeniería industrial de una universidad particular en Lima. [Tesis de Maestría, Universidad San Ignacio de Loyola]. Repositorio de la Universidad San Ignacio de Loyola. http://repositorio.usil. edu.pe/bitstream/USIL/9132/1/2019 BernalPortilla.pdf

Briñol,P., Falces, C.y Becerra,A.(2007). Psicología Social. McGraw-Hill Interamericana.

Chara,P. y Olortegui, A. (2018). Factores asociados a la actitud hacia la investigación en estudiantes universitarios de enfermería. Revista de Investigación y Casos de Salud CASUS, 3(2), 83-88. 10.35626/casus.2.2018.73

Cabrera, J., Cruzado, C., Purizaca, N., López, R., Lajo, Y., Peña, E., Apolaya, M. y Vélez, C. (2011) Factores asociados con el nivel de conocimientos y la actitud hacia la investigación en estudiantes de medicina en Perú. Revista panamericana de la salud, 33(3), 166-173. https://scielosp.org/pdf/ rpsp/2013.v33n3/166-173/es

Cervantes, L., Bermúdez, L. y Pulido, V. (2019). Situación de la investigación y su desarrollo en el Perú: reflejo del estado actual de la universidad peruana. Pensamiento y Gestión, 46, [1-10. 46. DOI: $10.14482 /$ pege. 46.7615

Maguiña, C. (2013). ¿Por qué investigar en el Perú? Revista Cuerpo Médico, 6(3), 6-8. https://sisbib.unmsm.edu.pe/BVRevistas/ cuerpomedicohnaaa/v6n3 2013/pdf/ a01v6n3.pdf

De La Cruz, J. y Rodríguez, E. (2019). La investigación: Mas allá del ranking de las universidades. Revista de la Facultad de Medicina Humana, 19(1), 7-12. DOI: 10.25176/RFMH.v19.n1.1786

Gálvez, N., Gonzáles, Y., y Monsalve, M. (2019). Actitud hacia la investigación científica al final de la carrera de Enfermería en Perú. Universidad Señor de Sipán. Chiclayo, Perú. Revista Gaceta Médica Boliviana, 42(1), 3237. 10.47993/gmb.v42i1.51

Giraldo, U. (2010). Memorias Simposio Internacional, II Nacional de Investigación y VIII Versión del Premio a Investigadores Pablo
Oliveros Marmolejo. Bogotá, Colombia. https://1library.co/document/qmwr5rwzmemorias-simposio-internacional-nacionalinvestigacion-investigadores-oliverosmarmolejo.html

Guzmán, E. (2018). Actitud hacia la investigación cientifica y rendimiento académico en alumnos de la carrera profesional de Educación Física de la Universidad Nacional Mayor de San Marcos Lima-Perú. [Tesis de Maestría, Universidad Nacional de Educación Enrique Guzmán Valle]. Repositorio de la Universidad Nacional de Educación Enrique Guzmán Valle. https://repositorio. une.edu.pe/bitstream/handle/UNE/2867/ TM\%20CE-Du\%204345\%20R 1\%20 -\%20Ramirez $\% 20$ Barrantes\%20Enrique. pdf? sequence $=1 \&$ isAllowed $=y$

Congreso de la República (2014). Ley Universitaria $N^{\circ} 30220 . \quad$ https://leyes.congreso.gob.pe/ Documentos/Leyes/Textos/30220.pdf

Mercado, M (2017). Actitudes hacia la investigación en los estudiantes de la carrera de Medicina Humana de la Universidad Peruana Los Andes. Educación Médica, 20(1), 95-98. 10.1016/j.edumed.2017.10.012

Meza, V. (2019). Actitudes hacia la investigación y la estadística en los estudiantes de la Universidad Peruana Unión, 2018. [Tesis de Licenciatura, Universidad Peruana La Unión]. Repositorio de la Universidad Peruana La Unión. https://repositorio.upeu. edu.pe/bitstream/handle/20.500.12840/2149/ Vicente Tesis Maestro 2019. pdf? sequence $=4 \&$ isAllowed $=y$

Morris, G. y Maisto, A. (2005). Introducción a la psicología. Prentice Hall. Inc. Pearson Educación.

Motta, L. (2009). Módulo de psicología social. Bucaramanga - Colombia: Universidad Nacional Abierta y a Distancia. https:// docplayer.es/2560592-Modulo-academico401514-psicologia-social.html

Munive, O. (2018). Actitudes hacia la Investigación Científica y Satisfacción Laboral en Profesionales de la Saluddel Instituto Nacional de Ciencias Neurológicas. Lima- Perú. [Tesis de Maestría, Universidad César Vallejo]. Repositorio de la Universidad César Vallejo. https://repositorio.ucv.edu.pe/bitstream/ handle/20.500.12692/12784/Munive MOM. pdf? sequence $=1$ \&isAllowed $=\mathrm{y}$

Muñoz, C. (2011). Cómo elaborar y asesorar una investigación de tesis. Editorial Pearson. 
Olivera, E. (2020). Actitudes hacia la investigación de bachilleres en administración y psicología de una universidad peruana- agosto. Revista de Ciencias Sociales y Humanidades, 11, 7081. 10.37135/chk.002.11.05

Ortega, R.,Veloso, R. y Hansen, O (2018). Percepción y actitudes hacia la investigación científica. Revista de Investigación en Ciencias Sociales y Humanidades, 5(2), 101109. DOI: $10.30545 /$ academo.2018.jul-dic. 2

Palomino, J. (2017). El portafolio, recurso didáctico para mejorar las actitudes hacia la investigación en estudiantes universitarios. [Tesis de Doctorado, Universidad Nacional de Educación Enrique Guzmán Valle]. Universidad Nacional de Educación Enrique Guzmán Valle. https://repositorio.une.edu. pe/bitstream/handle/UNE/1643/TD\%20\%20 CE\%201638P1\%20-\%20Palomino\%20Leon. pdf? sequence $=1 \&$ isAllowed $=y$

Paredes, F y Moreta, R. (2020). Actitudes hacia la investigación y autorregulación del aprendizaje en los estudiantes universitarios. CienciáMerica, 9(3), [1-16]. 10.33210/ ca.v9i3.263

Quispe, M. (2014). Actitudes de los internos de enfermería hacia la investigación y la elaboración de tesis para titularse. Universidad Nacional Mayor de San Marcos. [Tesis de Licenciatura, Universidad Nacional Mayor de San Marcos]. Repositorio de la Universidad Nacional Mayor de San Marcos. https://cybertesis.unmsm.edu.pe/bitstream/ handle/20.500.12672/4058/Quispe cm.pdf? sequence $=1 \&$ is Allowed $=y$

Rodríguez, R. (2017). Actitudes hacia la investigación en los participantes de postgrado de la universidad nacional abierta. Gestión, 2(1), 29-54. https://dialnet.unirioja. es/descarga/articulo/7467975.pdf

Rojas, M. y Méndez, R. (2013). Como enseñar a investigar. Un reto para la pedagogía universitaria. Educación y Ciencia, 16(1), 95-108. http://www.scielo.org.co/pdf/eded/ v16n1/v16n1a06.pdf

Rojas, H., Méndez, R. y Rodriguez, A. (2012). Índice de actitud hacia la investigación en estudiantes del nivel de pregrado. Entramado, 16, 216-219. http://www.scielo.org.co/pdf/ entra/v8n2/v8n2a15.pdf

Ruiz, C., Torres, V. (2005). La enseñanza de la investigación en la universidad: el caso de una universidad pública venezolana. Investigación $y$ Postgrado, 20(2), 14-34. https://dialnet. unirioja.es/servlet/articulo? codigo $=2310189$

Ruiz, J. (2010). Importancia de la investigación. Revista Cientifica, 20(2), 125-126. https:// www.redalyc.org/pdf/959/95912322001.pdf

Science Journal Ranking, Scimago Journal \& Country Rank (2018). Informe de SJR Scimago Journal \& Country Rank. https://www.scimagojr. com/countryrank.php?region $=$ Latin $\% 20$ America\&year $=20 \% 2018$

Valverde, M. (2005). Actitud de las enfermeras hacia la investigación y factores que intervienen en su realización en el Hospital Nacional Daniel Alcides Carrión. [Tesis de Licenciatura, Universidad Nacional Mayor de San Marcos]. Repositorio de la Universidad Nacional Mayor de San Marcos. https://cybertesis.unmsm.edu.pe/bitstream/ handle/20.500.12672/1052/Valverde cm.pdf? sequence $=1 \&$ isAllowed $=y$

Vázquez, A. y Manassero, M. (2008). El declive de las actitudes hacia la ciencia de los estudiantes: Un indicador inquietante para la educación científica. Revista Eureka sobre Enseñanza y Divulgación de las Ciencias, 5(3), 274-292. https://revistas.uca.es/index. php/eureka/article/view/3740/3317

Veliz, C. (2019). Las actitudes hacia la investigación y la disposición para la realización de la tesis en estudiantes de postgrado de dos universidades de Lima 2017. [Tesis de Doctorado, Universidad Nacional de Educación Enrique Guzmán y Valle]. Repositorio de la Universidad Nacional Enrique Guzmán y Valle. https:// repositorio.une.edu.pe/bitstream/handle/ UNE/2719/TD $\% 20 \mathrm{CE} \% 202024 \% 20 \mathrm{~V} 1 \% 20$ -\%20Veliz $\% 20$ Manrique $\% 20$ Cesar $\% 20$ David.pdf? sequence $=1$ \&isAllowed $=\mathrm{y}$ 